\title{
Septic Shock
}

National Cancer Institute

\section{Source}

National Cancer Institute. Septic Shock. NCI Thesaurus. Code C35018.

A state of acute circulatory failure characterized by persistent arterial hypotension despite adequate fluid resuscitation or by tissue hypoperfusion unexplained by other causes. 